\title{
Rassegna
}

\section{Sicurezza nella scelta dell'Inibitore di Pompa Protonica nel nefropatico cronico}

\author{
M. J. Sequenza ${ }^{1}$, M.R. Loi ${ }^{2}$, F. Londrino ${ }^{3}$, P. Sale ${ }^{4}$, S. Andrulli' ${ }^{5}$ A. Noce ${ }^{6}$, \\ O. Durante ${ }^{6}$, P. Zamboli ${ }^{7}$, F. Floccari ${ }^{8}$, F. Fiorini ${ }^{9}$, A. D’Amelio ${ }^{10}$, Luca di Lullo ${ }^{11}$, \\ A. Granata ${ }^{12}$, A. Mudoni ${ }^{13}$, F. Logias ${ }^{14}$
}

${ }^{1}$ Farmacia territoriale di Atzara (NU)

${ }^{2}$ U.O.S. Nefrologia e Dialisi, P.O. San Camillo Sorgono, Nuoro

${ }^{3}$ U.O.C. Nefrologia e Dialisi, P.O. Sant'Andrea, La Spezia

${ }^{4}$ U.O.C. Chirurgia, P.O. San Camillo Sorgono, Nuoro

${ }^{5}$ U.O.C. Nefrologia e Dialisi, P.O. A. Manzoni Lecco

${ }^{6}$ Università di Tor Vergata Roma, Roma

${ }^{7}$ Università Federico II Napoli, Napoli

${ }^{8}$ U.O.C. Nefrologia e Dialisi, P.O. Civitavecchia (Roma)

${ }^{9}$ U.O.C. Nefrologia e Dialisi, P.O. Rovigo

${ }^{10}$ U.O.C. Nefrologia e Dialisi, P.O. V. Fazzi, Lecce

${ }^{11}$ U.O.C. Nefrologia e Dialisi, P.O. Colleferro (Roma)

${ }^{12}$ U.O.C. Nefrologia e Dialisi, P.O. Agrigento

${ }^{13}$ U.O.C. Nefrologia e Dialisi, P.O. Tricase (LE)

${ }^{14}$ U.O.C. Nefrologia e Dialisi, Ospedale San Francesco, Nuoro

PPIs in Chronic Nephropatic Patient

ABSTRACT. Chronic nephropathic patients often present morphological and functional alterations of the gastro-enteric apparatus. Gastrointestinal diseases represent the most common and early signs. PPIs presented for many decades an important inhibitory effect on gastric acid secretion: they have a common chemical structure, a common mechanism of action and they are very important for treating acid-related pathologies, for Helicobacter Pylori eradication, for preventing and curing NSAIDs gastropathies. We prescribe this class of drugs to our patients even after the pathology resolution (gastroprotection). However can PPIs be indiscriminately used with chronic nephropathic patients or is it necessary to know the drug profile, in order to make a better choice? In this paper we argue that collateral effects are not very relevant and that they are very similar: their long-term usage is very safe. Power and efficacy of the different PPIs results very similar on the basis of how many milligrams of substance has been used and on the basis of clinical trials. The only exception that is shown in this paper is represented by 6 haemodialysis patients, which have been treated with lansoprazol (15 mgs). They presented gastritis and peptic ulcers, which were complicated by episodes of severe hematemesis and melena, with a consequent anaemia. Even though all PPIs have shown a similar clinical efficacy it is important to evaluate from time to time the (relative) advantages of each PPI. Criteria for a correct choice should be based mainly on official indications, on available doses and on the safety of the drug.

KeY WorDS. PPIs efficacy, Acid secretion, Drug tolerance

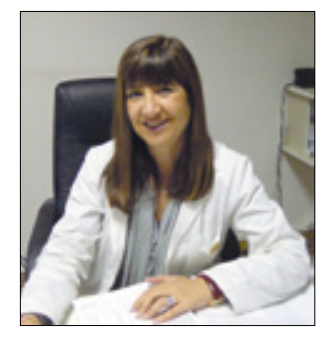

Maria Jose Sequenza

\section{Introduzione}

Il paziente affetto da nefropatia cronica facilmente presenta alterazioni morfologiche e funzionali dell'apparato gastro-enterico. I segni più comuni e precoci nella sindrome uremica cronica sono rappresentati, infatti, dai disturbi gastrointestinali. L'ammoniaca ha un notevole potere irritativo sulla mucosa gastro-enterica. Infatti la concentrazione dell'urea, oltre che ad aumentare nel sangue, è molto elevata sia nella saliva che nelle altre secrezioni del tubo digerente, dove per l'intervento di microrganismi va incontro a un processo di fermentazione ammoniacale (1). Il paziente nefropatico cronico sviluppa con estrema facilità gastriti 
e ulcere, di conseguenza sono abbastanza facili episodi di ematemesi e melena (2). La barriera gastrica alterata dal meccanismo di retrodiffusione degli idrogenioni risulta in un perenne stato di irritazione. Altri fattori concorrono a determinare gastropatia, fra questi si distinguono: la diatesi emorragica, la alterazione morfologica delle arteriole della sottomucosa, il deficit di vitamina D, l'iperparatiroidismo (3). Da alcuni decenni abbiamo a disposizione dei farmaci con potente azione inibente la secrezione acida gastrica: gli inibitori di pompa protonica (IPP). Quotidianamente somministriamo ai nostri pazienti questa classe di farmaci oltretutto con terapie che ininterrottamente continuano nel tempo nonostante la risoluzione della malattia (gastroprotezione). Ma gli IPP possono essere utilizzati tutti indistintamente nei pazienti nefropatici cronici oppure sarebbe utile conoscere il profilo del farmaco per una corretta scelta?

\section{Inibitori della pompa protonica}

Gli IPP, farmaci con potente azione inibente la secrezione acida gastrica, costituiscono una delle maggiori innovazioni farmacologiche che ha fortemente influenzato la gestione e la conoscenza delle patologie legate alla secrezione acida a livello gastrico.

Queste patologie comprendono la malattia da reflusso gastroesofageo (MRGE), la sindrome di Zollinger-Ellison, l'ulcera peptica benigna dello stomaco e del duodeno e le ulcere provocate dall'utilizzo a lungo termine di farmaci antinfiammatori non steroidei (FANS).

Gli IPP, inibendo la funzione dell'enzima ciclossigenasi1 (COX 1), hanno come effetto collaterale una riduzione della sintesi di prostaglandine, processo che dipende dallo stesso enzima.

Poiché tra le funzioni delle prostaglandine c'è la protezione della mucosa gastrica dall'acidità, si utilizzano gli IPP al fine di ridurre l'acidità e proteggere la mucosa stessa.

Gli IPP in commercio oggi in Italia sono cinque: il capostipite omeprazolo (immesso nel 1989), il lansoprazolo (1995), il pantoprazolo (1997), il rabeprazolo (1999) e l'esomeprazolo (2002). Sono disponibili a diverso dosaggio, a diverso costo per confezione e non tutte le indicazioni previste in scheda tecnica sono simili per i vari IPP e non tutti sono ammessi a rimborsabilità secondo la nota 1 CUF.

Il consumo di questi farmaci è cresciuto notevolmente negli ultimi anni e il motivo che ha causato questa crescita è da ricercare nell'estensione delle indicazioni secondo le modifiche delle Note AIFA $n^{\circ} 1$ e $n^{\circ} 48$.

Sebbene l'efficacia e la sicurezza fra i vari IPP sia sostanzialmente sovrapponibile, il costo è molto diverso.
Il lansoprazolo, divenuto farmaco equivalente, è stato inizialmente imposto come prima scelta da diverse regioni Italiane tra le quali la Sardegna.

\section{Meccanismo d'azione}

Gli IPP vengono riconosciuti come i farmaci più efficaci nell'inibizione prolungata della secrezione acida in quanto formano un legame irreversibile con la pompa protonica $\left(\mathrm{H}^{+} / \mathrm{K}^{+}\right.$ATPasi gastrica) e, agendo sul meccanismo finale di escrezione, rendono la cellula parietale insensibile ai diversi stimoli (colinergici, istaminergici, ormonali) al contrario dei farmaci $\mathrm{H}_{2}$.

L'omeprazolo, il lansoprazolo, il rabeprazolo, il pantoprazolo e l'esomeprazolo sono degli alpha-piridil-metil-sulfinil-benzimidazoli; si tratta di profarmaci la cui attivazione richiede un ambiente acido. Questi agenti raggiungono la cellula parietale dal circolo ematico ed essendo basi deboli si accumulano nei canalicoli secretori dove sono attivati mediante un processo catalitico mediato dai protoni, che porta alla formazione di una sulfenamide con particolare affinità per i gruppi -SH presenti sull'enzima.

Questa forma attiva reagisce mediante un legame covalente con i gruppi sulfidrilici della cisteina nel dominio extracellulare dell' $\mathrm{H}^{+} / \mathrm{K}^{+}$ATPasi. In particolare, il legame con la cisteina in posizione 813 è essenziale per l'inibizione della secrezione acida, che è un processo irreversibile. La pompa enzimatica viene risintetizzata, ma ciò richiede mediamente $72-90$ ore e questo spiega la lunga durata d'azione dei farmaci IPP.

Si calcola che nell'uomo il meccanismo di risintesi delle pompe enzimatiche sia in grado di riattivare $1 / 3$ della capacità acido-secernente delle cellule parietali entro le prime 24 ore dalla somministrazione del farmaco e che sono necessari circa tre giorni per tornare ai livelli iniziali di secrezione acida dopo la sospensione degli IPP. L'omeprazolo inibisce in modo selettivo anche l'anidrasi carbonica della mucosa gastrica, che a sua volta contribuisce alle sue proprietà acido-soppressive.

Diversi sono i meccanismi attraverso i quali gli IPP concorrono nell'eradicazione dell'Helicobacter Pylori: la marcata inibizione della secrezione acida gastrica favorisce l'accumulo dello ione ammonio, prodotto dal metabolismo dell'urea, con progressiva alcalinizzazione dell'ambiente fino a valori incompatibili con la sopravvivenza del germe.

L'innalzamento del pH gastrico e la riduzione delle secrezioni acide determinano una maggiore concentrazione ed efficacia degli antibiotici con un miglioramento della risposta immunitaria; infine effetti antimicrobici diretti sono mediati dall'inibizione di vari enzimi batterici. Pur se il meccanismo patogenetico del danno da FANS 
è rappresentato dalla ridotta sintesi di prostaglandine gastroprotettrici, l'acidità gastrica potenzia notevolmente il danno mucosale indotto dai FANS e da questo deriva il razionale dell'uso degli antisecretori a scopo preventivo. Gli IPP si sono dimostrati farmaci insostituibili nel trattamento della MRGE, che è una malattia cronica con tendenza alle recidive che sono efficacemente prevenute dagli IPP con un trattamento a lungo termine che va effettuato in varie modalità (continuativo, intermittente, al bisogno) in relazione alla gravità del quadro clinico e al differente andamento della malattia nel singolo paziente.

È ancora molto diffuso nella classe medica il timore del reflusso acido notturno (da cui la prescrizione serale dell'antisecretore) ma è ormai dimostrato che gli episodi di reflusso sono molto più frequenti di giorno e quindi è più razionale somministrare gli IPP al mattino. Nei casi resistenti di MRGE in cui si raddoppiano i dosaggi è consigliata la doppia somministrazione (mattina e sera).

\section{Farmacocinetica}

Gli IPP sono dei composti acido-labili. Le formulazioni orali (a rilascio ritardato) sono composte da granuli gastroprotetti racchiusi in una capsula di gelatina (omeprazolo e lansoprazolo) o da compresse gastroprotette (rabeprazolo e pantoprazolo).

I granuli si dissolvono solo a $\mathrm{pH}$ alcalino, prevenendo in tal modo l'inattivazione dei farmaci da parte del succo acido nell'esofago e nello stomaco.

Gli IPP sono assorbiti rapidamente, legati largamente a proteine, e metabolizzati ampiamente nel fegato da due enzimi del sistema del citocromo P450, in particolare CYP2C19 (mefentoinaidrossilasi) e CYP3A4 (nifedipinaidrossilasi).

Il pantoprazolo è metabolizzato anche da una sulfotransferasi citosolica e questo spiegherebbe la minore interazione con farmaci che usano il Citocromo P450 per la metabolizzazione (4). I loro metaboliti solfati vengono escreti con le feci e con le urine. La loro emivita plasmatica è di circa 1-2 ore, ma la loro durata di azione può protrarsi più a lungo. È stata accertata l'esistenza di polimorfismo genetico del citocromo P450 2C19 coinvolto nel metabolismo degli inibitori di pompa protonica: nell'eradicazione dell'Helicobacter Pylori, una più bassa percentuale di eradicazione è stata osservata nei soggetti metabolizzatori rapidi che assumono lansoprazolo oppure omeprazolo, ma non col rabeprazolo.

Le percentuali di guarigione dell'esofagite sono risultate più basse nei metabolizzatori rapidi con lansoprazolo ma non con rabeprazolo (5).

Tra i diversi IPP esistono differenze farmacocinetiche (biodisponibilità, emivita di eliminazione, metabolismo), con un differente impatto sulla risposta clinica.

La biodisponibilità del rabeprazolo nel primo giorno di somministrazione è superiore a quella degli altri IPP. Rabeprazolo, lansoprazolo, pantoprazolo presentano biodisponibilità simili al giorno 1 e 5 ; la biodisponibilità di omeprazolo aumenta di 1.5-2 volte al quinto giorno e la biodisponibilità di esomeprazolo aumenta di 3 volte sempre al quinto giorno.

L'esomeprazolo determina un controllo della secrezione acida più efficace e prolungato rispetto agli altri inibitori della pompa protonica (5).

Il tenatoprazolo (non disponibile in Italia) è un nuovo inibitore di pompa protonica che ha un'emivita di 5-7 volte più lunga, rispetto agli attuali IPP.

Sembra che nei pazienti con insufficienza renale cronica e cirrosi epatica, una singola dose giornaliera di IPP non determini un loro evidente fenomeno di accumulo. La patologia epatica riduce sensibilmente la clearance del lansoprazolo e la riduzione del dosaggio va considerata nelle gravi epatopatie.

\section{Effetti indesiderati}

Gli IPP sono una classe di farmaci ben tollerata. Gli eventi avversi più comuni, mal di testa, diarrea, nausea, vomito, dolori addominali, flatulenza, costipazione, sonnolenza, alterazioni del sonno, vertigini, sono stati riportati in meno del 5\% dei pazienti trattati con lansoprazolo e omeprazolo. La frequenza di questi eventi avversi, con i due inibitori di pompa protonica, è paragonabile a quello del placebo e degli antagonisti del recettore $\mathrm{H}_{2}$ In certi casi gli effetti indesiderati spariscono con l'interruzione del trattamento.

Gli IPP possono provocare nefrite interstiziale acuta (AIN). Il primo caso di AIN descritto in letteratura (1992) fu indotta dall'omeprazolo (6). Ne sono seguite numerose. Nel 2004 sono stati segnalati dei casi di AIN indotti da pantoprazolo, rabeprazolo e da lansoprazolo nel Regno Unito.

Tali evidenze sono convalidate da altri 18 casi di AIN indotti da IPP, confermate da esame bioptico, in una clinica in Nuova Zelanda. Il meccanismo con il quale si sviluppa l'AIN farmaco-indotta non è conosciuto, ma si sospetta sia su base immunologica $(7,8)$.

Su JAMA (9) è stato riportato un ampio studio osservazionale che rileva una possibile associazione fra l'uso cronico degli IPP e l'aumento di fratture osteoporotiche di femore sulla base fisiopatologica di ridotto assorbimento di calcio.

Le associazioni di fratture di anca, polso, avambraccio e altri siti appaiono deboli e solo leggermente più alto 
rispetto ai rischi in popolazioni di controllo abbinati per età. Essi possono aumentare col trattamento degli IPP, ma probabilmente lo fanno solo negli individui in cui sono presenti anche altri fattori di rischio (fumo, alcol, cattiva alimentazione, steroidi ecc.) (10).

È stato segnalato un aumento della prevalenza di polmoniti e di enteriti da Campylobacter cosi come il raddoppio del rischio di infezioni da Clostridium difficile in soggetti trattati con IPP in ambiente ospedaliero e in reparti di terapia intensiva.

Sono segnalate manifestazioni diarroiche, da mettere in relazione con forme di colite microscopica in corso di trattamento con lansoprazolo.

È stata dimostrata un'interazione farmacologica tra clopidogrel e IPP a livello del citocromo CYP2C19. Il clopidogrel, che è un pro-farmaco, è attivato dal CYP2C19. Anche alcuni IPP (specialmente omeprazolo, ma non pantoprazolo) sono trasformati dallo stesso citocromo ed esercitano un effetto inibitorio che interferisce negativamente con l'attivazione del clopidogrel e con la sua attività antiaggregante piastrinica.

Dato che il clopidogrel e gli IPP hanno un'emivita inferiore a due ore, l'interazione farmacocinetica potrebbe essere ridotta ai minimi termini distanziando la loro assunzione, ad esempio, facendo assumere l'IPP al mattino e il clopidogrel la sera.

La ridotta secrezione di acido gastrico e pepsina determinata dagli IPP può ridurre l'assorbimento della vitamina B12 alimentare legata alle proteine, ma non la vitamina B12 degli integratori. L'acido gastrico è necessario per distaccare la vitamina B12 dalle proteine per l'assorbimento.

Ridotti livelli di vitamina B12 possono essere più comuni con gli IPP che con gli H2-bloccanti, perché è più probabile che essi determinino acloridria (completa assenza della secrezione di acido gastrico). Comunque, un deficit clinicamente significativo di vitamina B12 è improbabile, a meno che la terapia con gli IPP sia prolungata (due anni o più) o l' introito della vitamina alimentare sia basso.

I livelli di vitamina B12 dovrebbero essere controllati in persone che assumono alte dose di IPP per periodi prolungati.

L'uso degli inibitori della pompa protonica in gravidanza avviene per il trattamento della malattia da reflusso gastroesofageo. Da studi clinici in vivo, riguardanti l'assunzione degli IPP durante i primi mesi di gravidanza, se somministrati alle dosi consigliate, non è emerso alcun effetto avverso sul feto o tossicità-peri-postnatale (11). Da uno studio sul ratto si è visto che gli IPP vengono escreti nel latte materno fino a un valore che non supera lo $0.02 \%$ della dose assunta. Si è anche visto che il farma- co passa la placenta e che tale passaggio va intensificandosi con il progredire della gravidanza; comunque sia non si dispone di dati certi per l'uomo.

Pertanto, prima di iniziare la terapia con gli IPP è consigliabile tenere conto dei vantaggi del trattamento per la madre e degli eventuali rischi che tale trattamento può comportare per il feto.

La co-somministrazione di digossina e lansoprazolo può portare a un aumento dei livelli plasmatici di digossina. Pertanto nei pazienti che ricevono digossina i livelli plasmatici devono essere monitorati e se necessario la dose di digossina deve essere aggiustata.

Tra il 2003 e il 2008 l'Assessorato alla Sanità della Sardegna, ma anche di altre regioni d'Italia, limitava la rimborsabilità al solo lansoprazolo, per cui risultava l'unico farmaco ad essere dispensato dalle farmacie ospedaliere. Nell'arco di un anno presso l'Unità Operativa di Dialisi del Centro San Camillo di Sorgono abbiamo osservato 6 casi di gastriti e ulcere peptiche su 30 pazienti in trattamento emodialitico, in alcuni casi complicate da gravi episodi di ematemesi e melena, tutti documentati da esame endoscopico. Tutti i 6 pazienti assumevano $15 \mathrm{mg}$ di lansoprazolo come gastroprotettore. L'esame gastroscopico eseguito su questi pazienti era caratterizzato da mucosa antrale iperemica, piloro spastico ed ematoso con erusioni confluenti che interessavano l'80\% della cavità. Successivamente all'esame gastroscopico tutti i pazienti hanno intrapreso il trattamento con $40 \mathrm{mg}$ di esomeprazolo per tre mesi e, al successivo controllo endoscopico, si è osservata la completa guarigione della mucosa gastrica.

\section{Conclusioni}

Gli IPP hanno una struttura chimica affine e uno stesso meccanismo d'azione, con differenze farmacocinetiche, farmacodinamiche e metaboliche (la differente metabolizzazione epatica attraverso il citocromo P450 influenza l'interazione con altri farmaci).

Gli IPP sono farmaci molto importanti per il trattamento delle patologie acido correlate, per l'eradicazione dell'Helicobacter Pylori, per la prevenzione e la cura della gastropatia da FANS. I loro effetti collaterali non sono molto rilevanti ed abbastanza simili, il loro impiego nel lungo termine è sicuro.

La potenza e l'efficacia dei vari IPP, dall'analisi comparativa dei vari trial clinici, risulta essere molto simile sulla base dei milligrammi di sostanza utilizzata.

Premesso che tutti gli IPP hanno dimostrato una efficacia clinica sovrapponibile, vanno valutati di volta in volta i vantaggi (relativi) di ciascun IPP (lunga esperienza clinica dell'omeprazolo, maggior rapidità nel risolvere $\mathrm{i}$ 
sintomi del lansoprazolo, minori interazioni farmacologiche del pantoprazolo, maggiore rapidità d'azione del raboprazolo, maggior controllo della secrezione acida dell'esomeprazolo).

I criteri di scelta di un IPP sembrano quindi basati, principalmente sulle indicazioni autorizzate, sulle formulazioni disponibili, sul profilo di sicurezza del farmaco.

\section{Riassunto}

Il paziente nefropatico cronico facilmente presenta alterazioni morfologiche e funzionali dell'apparato gastroenterico. I segni più comuni e precoci nella sindrome uremica cronica sono rappresentati dai disturbi gastrointestinali.

Da alcuni decenni abbiamo a disposizione dei farmaci con potente azione inibente la secrezione acida gastrica: gli inibitori di pompa protonica (IPP) hanno una struttura chimica affine, uno stesso meccanismo d'azione e sono molto importanti per il trattamento delle patologie acido correlate, per l'eradicazione dell'Helicobacter Pylori, per la prevenzione e la cura della gastropatia da farmaci antinfiammatori non steroidei (FANS). Somministriamo ai nostri pazienti questa classe di farmaci, con terapie che continuano nel tempo, nonostante la risoluzione della malattia (gastroprotezione). Ma gli IPP possono essere utilizzati indistintamente nei nefropatici cronici oppure sarebbe utile conoscere il profilo del farmaco per una corretta scelta?

In questo articolo si argomenta che i loro effetti collaterali non sono molto rilevanti e sono abbastanza simili: il loro impiego nel lungo termine è sicuro. La potenza e l'efficacia dei vari IPP, dall'analisi comparativa dei vari trial clinici, risulta essere molto simile sulla base dei milligrammi di sostanza utilizzata. Lunica eccezione illustrata in questo lavoro è rappresentata da 6 pazienti in emodialisi, trattati con lansoprazolo (15 mg), che presentavano gastriti e ulcere peptiche complicate da gravi episodi di ematemesi e melena con conseguente anemia.

Tutti gli IPP hanno dimostrato un'efficacia clinica sovrapponibile, tuttavia vanno valutati di volta in volta $\mathrm{i}$ vantaggi (relativi) di ciascun IPP. I criteri di scelta di un IPP sembrano basati, principalmente sulle indicazioni autorizzate, sulle formulazioni disponibili, sul profilo di sicurezza del farmaco.

Parole Chiave. Efficacia IPP, Secrezione acida, Tollerabilità
Indirizzo degli Autori:

Dr.ssa Maria Jose Sequenza

Farmacia territoriale

via Vittorio Emanuele

Atzara $08030(\mathrm{Nu})$

msequenza@tiscali.it

Dott.ssa Maria Rita Loi

U.O.S. Nefrologia e Dialisi, P.O. San Camillo

via IV Novembre

08038 Sorgono $(\mathrm{Nu})$

loi.ri@tiscali.it

\section{Bibliografia}

1. Zelbenick EB, Goyal RK. Gastrointestinal manifestation of chronic renal failure. Sem Nephrol, New York : Grume \&Straton, 1981.

2. Andrulli A, Malfi B, Recchia S, et al. Patients with chronic renal failure are not at risk of developing chronic peptic ulcers. Clin Nephrol 1985; 23: 245.

3. Milito G, Taccone-Galluci M, Brancaleone CV, et al. The upper and lower gastrointestinal tract in uremic patients on long-term hemodialysis. Kidney Int 1985; 28(Suppl17): S157-S160.

4. Bensancon M, Simon A, Sachs G, Shin JM. Sites of reaction of the gastric H, K-ATPase whit extracytoplasmic thiol reagents. J Biol Chem 1997; 272: 22438-46.

5. Fock KM, Ang TL, Bee LC, Lee EJ. Proton pump inhibitors: do differences in pharmacokinetics translate into differences in clinical outcomes? Clin Pharmacokinet 2008; 47: 1-6.

6. Ruffenach SJ, Siskind MS, Lien YH. Acute interstitial nephritis due to omeprazole. Am J Med 1992; 93: 472-3.

7. Ra A, Tobe SW. Acute interstitial nephritis due to pantoprazole. Ann Pharmacother 2004; 38 41-5.

8. Geevasinga N, Coleman PL, Roger SD. Rabeprazole-induced acute interstitial nephritis. Nephrology (Carlton) 2005; 10: 7-9.

9. Yang YX, Lewis JD, Epstein S, Metz DC. Long-term proton pump inhibitor therapy and risk of hip fracture. JAMA 2006; 296; 2947-53.

10. Current Opinion in Gastroenterology. 2010, 26 (6); 62431.

11. Nikfar S, Abdollahi M, Moretti ME. Use of proton pump inhibitors during pregnancy and rates of major malformations: a metanalysis. Dig Dis Sci 2002; 47: 1526-9. 